\title{
La CONSTRUCCIÓN SOCIAL DE DIFICULTADES DE APRENDIZAJE EN LAS PRÁCTICAS EDUCATIVAS
}

THE SOCIAL CONSTRUCTION OF LEARNING DISABILITIES ACROSS EDUCATIONAL PRACTICES

Soraya SÁ NCHEZ ${ }^{1}$

RESUMEN : de acuerdo con los sistemas de clasificación y los procesos de tipificación, la construcción social de categorías y la concepción transaccional que asumimos en la comprensión de las Dificultades de A prendizaje, entendemos que éstas no son un hecho objetivo, sino un constructo cultural mente determinado, interpretado y explicado por una comunidad, en un contexto educativo, sociocultural e histórico determinado por la práctica discursiva de las dificultades de aprendizaje.

PALABRAS-CLAVE: construcción social; dificultades de Aprendizaje; categoría; prácticas discursivas; educación especial.

A BSTRACT: according to the systems of classification in human cultures, the social construction of categories and the transactional conception that we assume in comprehending Learning Disabilities, we understand that these are not an objective fact, but rather a cultural construct, interpreted and explained by a community in a specific educational, sociocultural and historical context determined by the discursive practice of learning disability.

KEYWORDS: social construction; learning disabilities; category, discursive practices; special education.

\section{La construcción de las categorías sociales (Construction of social categories)}

A través de la reconceptualización de las Dificultades de A prendizaje desde una perspectiva sociocultural, pretendemos avanzar en la compresión del complejo entramado de relaciones que subyace a la aparición de la categoría "Dificultades deA prendizaje" y las restricciones que implica para la participación y en la percepción de la competencia, el éxito y el fracaso escolar.

\footnotetext{
${ }^{1}$ Universidad de Alcalá, España - Departamento de Psicopedagogía y Educación Física. Área de Psicología Evolutiva y de la Educación. Universidad de Alcalá (Madrid, España) - Soraya Sánchez Valverde. sorayasv@yahoo.es.
} 
Para entender los procesos por los que se crean las categorías sociales y los significados que se les atribuyen es necesario adoptar una visión holística sobre cómo se construye el mundo social no solo como realidad o "facticidad objetiva" sino también como "conjunto de significados subjetivos" queseexpresan en la actividad que desarrollan las sociedades humanas (BERGER; LUCKMAN N, 1968). Dicha visión, implica considerar estas dos dimensiones como entidades interdependientes que confluyen constituyendo la naturaleza dual de la realidad social. Estos dos conceptos clave, constituyen nuestro punto de partida para el estudio de la construcción social de la realidad y, con ello, de la formación de la categoría "Dificultades de Aprendizaje”.

Los procesos por los que los significados subjetivos atribuidos a esta categoría se convierten en hechos culturalmente aceptados y compartidos por una comunidad se generan mediante la participación activa de los individuos en la construcción de la realidad social a través de sus interacciones en la vida cotidiana y por medio de los significados subjetivos que construyen acerca de ella (RITZER, 1993; SCHÜTZ, 1993). Dichos significados y el sentido quelas personas otorgan a la realidad explican sus acciones sociales, por tanto, acceder a el los es desuma importancia para intervenir sobre las prácticas sociales que están determinando. Paradójicamente, este mundo construido socialmente se internaliza en la conciencia individual como una realidad objetiva (MEAD, cfr. BERGER; LUCKMAN N, 1968, p. 33).

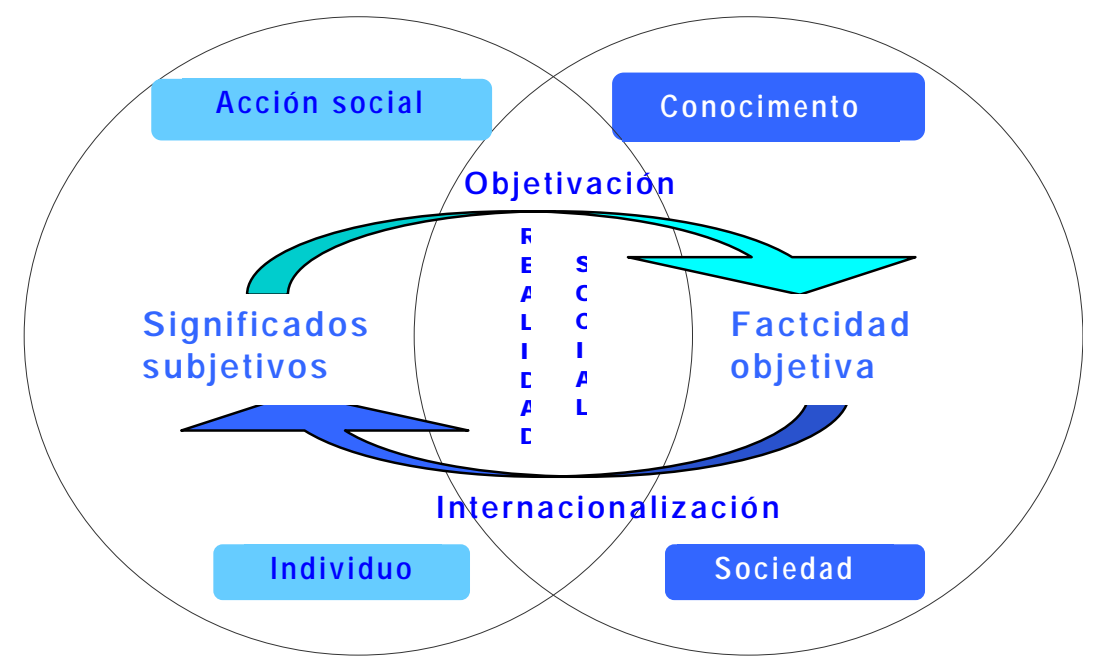

Figura 1. El carácter dual de la realidad social

La figura anterior refleja cómo ambas dimensiones se relacionan e influyen dialécticamente generando una interdependencia cultura-estructura. Esta interdependencia es de suma importancia para la evolución de las sociedades, ya que, si la estructura social no fuese susceptible a la acción de los 
sujetos, las estructuras sociales permanecerían fijas, y si no existiera un consenso de significados culturalmente establecido, no sería posi ble compartir la "realidad intersubjetiva" que nos permite convivir en sociedad (BERGER; LUCKMANN, 1968). La agencia de los individuos como reproductores y transformadores de la cultura, supone asumir que ésta es una construcción abierta constantemente a la negociación y al cambio, en permanente dinamismo (KLOTZ, 2003).

El modelo de "marcos de significado" propuesto por Stangvik (1998) describe las bases sociales de la objetivación o modos en que pueden hacerse objetivas la discapacidad y la desviación normativa mediante dicha negociación del significado. Los significados en torno a éstos y otros conceptos se construyen en diferentes marcos de conocimiento y niveles que se organizan formando una estructura jerárquica interrelacionada: "discurso general", "paradigma", "modelo", "teoría y metateoría” y "prácticas e instrumentos".

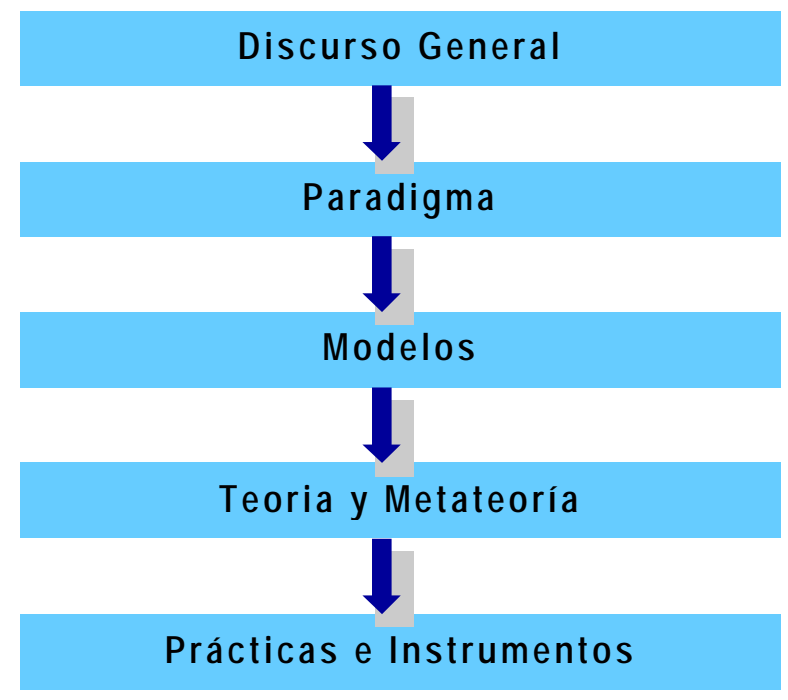

Figura 2. Marcos de significado (reproducido de Stangvik, 1998, p. 140)

El discurso general, al que más adelante nos referimos como "discurso formal" usando la terminología de Foucault (1976), suel e estar teñido de intereses políticos y profesionales, mientras que los discursos más micro en que puede subdividirse, "discursos informales" o "prácticas discursivas", reflejan los intereses de los participantes en un contexto. Ambos tienen en común que se construyen en torno a un conjunto de presupuestos quetamizan la información y condicionan la mirada con la que nos acercamos al mundo, nuestra perspectiva. Como investigadores, nuestra perspectiva seencuentra mediada por los sucesivos niveles de paradigmas, modelos, teoría y metateoría. 
Las prácticas e instrumentos son el último, y por ello, el nivel de mayor concreción del diagrama. Foucault (1982) las Ilama prácticas "divisorias" para referirse a este modo de objetivación haciendo hincapié en que el significado de un conjunto de prácticas e instrumentos, contextualiza y adscribe a una persona con discapacidad a un significado específico.

\subsection{TIPIFICAR Y CLASIFICAR A LOS INDIVIDUOS (TYPIFY ANd CATEgorize THE INDIVIDUAL)}

Las tipificaciones son procesos mentales que nos permiten simplificar la realidad para comprender, e intentar controlar, diferentes aspectos de nuestra experiencia en la vida cotidiana, de forma que el conjunto de tipificaciones y pautas de interacción que se establecen por medio de ellas no solo conforman la estructura social, sino que, según Schütz, participan de nuestro pensamiento del sentido común prevaleciendo como conocimiento establecido y socialmente aceptado en un determinado momento histórico y sociocultural.

Además de simplificar nuestra experiencia, gracias a las categorías podemos construir juicios acerca de los fenómenos. De acuerdo con la filosofía kantiana, éstas son formas "a priori" del pensamiento que nos permiten agrupar los fenómenos que comparten características comunes otorgándoles así unidad y significación. Al igual que las tipificaciones, no es posible encontrarlas empíricamente en la real idad "objetiva", ya que son nociones abstractas que nos sirven como instrumentos o "artefactos" para organizar la realidad. Desde una perspectiva sociocultural (WERTSCH, 1991, 1998), las categorías sirven como recursos mediadores, como "artefactos" culturales quejuegan un papel fundamental en el sentido que damos a nuestras experiencias.

Los artefactos, componentes de la cultura, son simultáneamente materiales e ideales/ simbólicos. Se materializan en forma de objetos, palabras, rituales y otras prácticas cultural es que median la vida humana (COLE, 1998, p. 292).

Como hemos visto, las categorías existen en el razonamiento de los individuos (como formas "a priori" de pensamiento), pero también están presentes entre las personas en la interacción social. En las interacciones "cara a cara" que tienen lugar en la vida cotidiana, utilizamos continuamente esquemas tipificadores para aprehender y relacionarnos con los otros. Cuando tipificamos a las personas reducimos sus atributos individuales a las características grupales estereotipadas (DÍAZ-AGUADO; MARTínEZ; MARTíN, 2004, p. 89), incluyéndolas en categorías sociales a las que les asignamos un determinado valor en función de la comparación social con otros grupos. De este modo, interpretamos determinados aspectos del comportamiento individual como resultado de esta tipificación y en correspondencia con la categoría que les asignamos. N o obstante, Berger y Luckmann (1968) afirman que estos esquemas son recíprocos y pueden negociarse en la interacción. 
Estas diferencias de val or o estatus que establecemos entre las personas, o entre unos grupos y otros, introducen dinámicas injustas de poder (HALL, 2000) que se mantienen mediante la creación de reglas no explícitas de funcionamiento grupal que determinan qué conductas constituyen infracciones o desviaciones de la norma (BECKER, 1963). Los estereotipos hacia determinados grupos pueden participar deesteproceso al funcionar como formas delegitimar y mantener dichas diferencias de estatus y poder, según muestra un estudio reciente sobre violencia entre iguales (DÍAZ-AGUADO; MARTÍNEZ; MARTÍN, 2004).

\section{El concepto de Dificultades de A prendizaje desde la perspectiva sociocultural (The Concept of Learning Disability from Sociocultural Perspective)}

Considerando los rasgos que comportan muchos de los paradigmas y modelos presentes en el ámbito de la Educación Especial y las Necesidades Educativas Especiales, pareceoportuno simplificar estas tendencias agrupándolas, siguiendo a Stangvik (1998, p.147), en dos aproximaciones generales a la discapacidad: "la discapacidad como deficiencia" y "la discapacidad como una práctica social negociada". Nos interesa aquí hacer especial hincapié en la segunda aproximación, según la cual "los problemas de las personas son considerados como social menteconstruidos y dependientes de dinámicas deinteracción social". Esta perspectiva no se sitúa en el extremo de la primera porque no obvia la trascendencia de las disfunciones individuales, sino que focaliza la atención en las consecuencias sociales y subjetivas de éstas en un contexto social particular.

Dentro delas corrientes depensamiento queentienden la discapacidad desde esta aproximación relacional, este autor destaca el concepto de "transacción" como elemento claveen la atribución del significado que seleda a las Dificultades de A prendizaje:

En el curso de la transacción con los entornos psicológico y social, las dificultades de aprendizaje son revestidas de significado. Este es un proceso de doble sentido en que la persona es tanto construida como constructora, y las transacciones son tanto interaccionales como interpretativas (STANGVIK, 1998, p. 148).

De acuerdo con esta comprensión transaccional, analizaremos a continuación los aspectos sociocul turales de la discapacidad, entendida ésta como un proceso evolutivo y de naturaleza dinámica, que sólo puede comprenderse en toda su complejidad si seexaminan los contextos de aprendizajey las interacciones que se establecen en sus miembros (GINDIS, 2003).

Asumir una perspectiva sociocultural en el estudio de las Dificultades de A prendizaje nos al eja de concepciones estáticas del déficit, del contexto y del aprendizaje, pero además, nos permite profundizar en el papel que desempeñan las estructuras de actividad en contextos específicos como determinantes del éxito 0 
fracaso escolar, en cuanto sistemas que pueden facilitar u obstaculizar las oportunidades de participación en las tareas y actividades que tienen lugar en ellas (GERGEN, 1990; GUTIÉRREZ, 2002; GUTIÉRREZ et al., 1999; M CDERMOTT, 1993; MCDERM OTT; VAREN NE, 1996; MCDERMOTT; VAREN NE, 1998; WEN GER, 2001).

De esta forma, las estructuras de actividad tienen un importante peso en la aparición de las Dificultades de Aprendizaje y una gran trascendencia tanto en el desarrollo de competencias necesarias para la interacción social como en la configuración de una determinada identidad social y estatus en la comunidad (GERGEN, 1990; WENGER, 2001).

Numerosos investigadores (DUDLEY-MARLING, 2004; GERGEN, 1990; GINDIS, 2003; GUTIÉRREZ, 2002; GUTIÉRREZ et al., 1999; MCDERM OTT, 1993; MCDERM OTT; VAREN NE, 1996; M CDERM OTT; VAREN NE, 1998; WENGER, 2001) sustentan la idea de que las Dificultades de Aprendizaje son una categoría culturalmente construida y estructuralmente organizada en contextos situados de actividad y, por tanto, no pueden localizarse ni atribuirse al individuo y sus capacidades deficitarias. Desde esta perspectiva no se pretende afirmar que las diferencias individuales no existan, sino que éstas interactúan recíprocamentecon las condiciones cultural es y organizativas del escenario donde se lleva a cabo la actividad, por medio de las prácticas y las relaciones interpersonales que se establecen entre los miembros de una comunidad. Es en el entorno social, por tanto, donde las diferencias se convierten en deficiencias, ya que son las implicaciones sociales de la discapacidad y no la discapacidad en sí misma la que produce los mayores problemas al restringir el acceso y la participación en las prácticas socioculturales, limitando el desarrollo y el aprendizaje del niño (GINDIS, 1995, 1999, 2003; RUEDA; GALLEGO; MOLL, 2000; VYGOTSKY, 1983, 1995).

Este argumento se enfatizó ya en la discusión acerca de las relaciones entre los conceptos de "discapacidad primaria" y "discapacidad secundaria" introducidos por Vygotsky (1995, cfr. GINDIS, 1999; 2003). El primero de ellos, hace alusión a las características personales asociadas a la discapacidad y a las limitaciones que se derivan de ellas, como pueden ser aquellas en el dominio de significados, en el aprendizaje de conocimientos y formas de relación social mente apropiadas. El segundo tipo de discapacidad se debe a factores sociales y constituye lo que se ha Ilamado la dimensión social de la discapacidad. Según Vygotsky, esta dimensión conlleva las repercusiones más graves para el desarrollo de las funciones psicológicas superiores. 


\subsection{La construcción sociocultural de las Dificultades de Aprendizaje (Sociocultural construction of Learning Disabilities)}

De acuerdo con los argumentos sobre la tipificación y la construcción de categorías, así como sobre la concepción transaccional, las D ificultades de A prendizaje, y por extensión, a otras categorías sociales de clasificación de los seres humanos, no son hechos objetivos, sino constructos histórica y culturalmente determinados, revestidos de significado social, que se objetivizan por medio de la actividad humana convirtiéndose en un conocimiento establecido y definido por una colectividad en un contexto educativo, histórico y sociocultural concreto.

Las aproximaciones transaccionales a la discapacidad ponen especial atención en la interacción y el control social en la construcción objetiva y subjetiva de la discapacidad (STANGVIK, 1998). Para llegar a comprender estas dos dimensiones de la discapacidad, es importante distinguir entre significados "subjetivos" y "objetivos". Recurrimos a la diferenciación realizada por SCHÜTZ (1993) según la cual los significados "subjetivos" son relativos a aquel los elementos de la realidad social que resultan significativos para el individuo, mientras que, Ios significados social es compartidos u "objetivos", son conjuntos de significados relevantes para una colectividad, que se plasman en la cultura del grupo y se comparten por todos sus miembros.

Para evitar ambigüedades, definiremos cultura como "conjunto de conceptos, símbolos y creencias compartidas" que se construyen, se hacen objetivos y se legitiman a partir de las interacciones cotidianas y de las prácticas que tienen lugar entre los miembros de una determinada comunidad en un marco concreto de estructuras socioculturales (MCDERMOTT; VARENNE, 1998, p. 27; SCHUTZ, 1993). No nos referimos, por tanto, a la cultura en su sentido más amplio, sino a la cultura "en su forma concreta, como práctica, como las experiencias sociales y materiales desarrolladas y acumuladas históricamente por los seres humanos" (MOLL, 1997, p. 45)

En la siguiente figura podemos observar cómo interactúan los cuatro dimensiones clave que venimos relacionando, a saber: Cultura, Estructura, Prácticas y Comunidad; en relación a la forma en que se perciben y se construyen las diferencias individuales. 


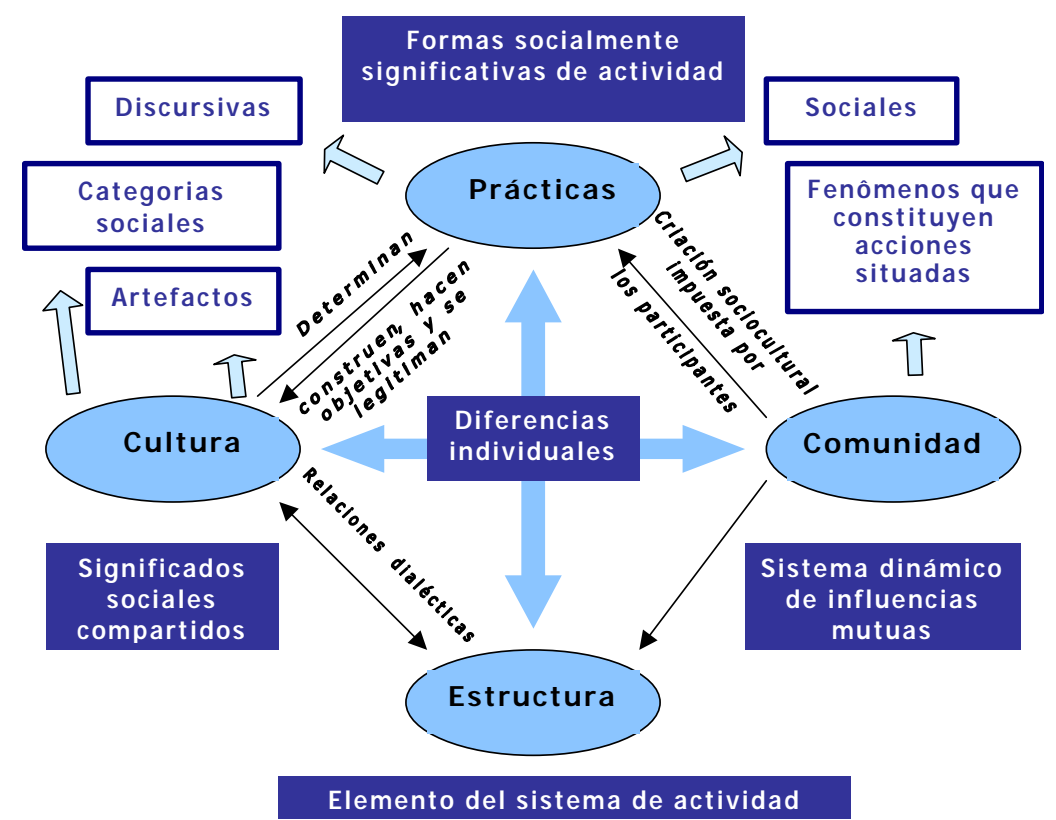

Figura 3 - Dimensiones relacionadas en la construcción de la diversidad

\section{La institucionalización de las Dificultades de A prendizaje (In stitutionalization of Learning Disabilities)}

Las Dificultades de Aprendizaje, así como cualquier otra categoría, deben entenderse en el contexto de la institución donde se crearon, ya que están interrelacionadas con este detal forma quelas categorías seintroducen en el repertorio de prácticas institucionales como recursos fundamentales en la toma de decisiones, en la organización de las oportunidades de enseñanza y el aprendizaje, en la dotación de recursos y en discurso sobre la vida escolar (HJÖRNE; SÄLJÖ, in press; MÄKITALO; SÄLJÖ, 2002).

El discurso del individualismo que preside la institución escolar no puede sino situar las Dificultades de A prendizaje en la cabeza de los alumnos, ya queequipara el aprendizajecon el dominio dehabilidades. Consecuentemente, un alumno con dificultades para aprender será aquel que no alcance un determinado nivel de dominio de las habilidades exigidas para un determinado momento, es decir, aquel que presente un déficit en ciertas habilidades significativas para la institución y no en otras (MCDERMOTT, 1993, p. 272; DUDLEY-MARLING, 2004, p. 484).

La designación institucional "Alumnos con Dificultades de A prendizaje" ofrece una explicación al fracaso escolar que mantiene la legitimidad y el status quo de la escuela al situar la responsabilidad del aprendizaje en los individuos 
concretos. Sin embargo, desde nuestra posición, consideramos que es la estructura y la cultura escolar la que en gran medida determina a priori quién fracasará y quién tendrá éxito porque el fracaso escolar es una "patología organizativa" (SKRTIC, 1995), es decir, que de algún modo responde a "procesos y estructuras que son de algún modo endémicas a la finalidad educativa y que no pueden ser invertidas, reestructuradas y deconstruidas" (CLARK; DYSON; MILLWARD, 1998, p. 167).

La institución escolar se rige por un discurso o sistema de reglas conformado históricamente y basado fundamentalmente en la medicina y la psicología. Las prácticas que tienen lugar en este contexto se establecen en torno a unos objetivos y una estructura de participación determinados, que se organizan de manera coherente con dichos model os normativos, y que a su vez, generan ciertos criterios diagnósticos y de competencia a partir de los cuales se conceptualiza y justifica la presencia o ausencia de las Dificultades en el Aprendizaje.

\begin{abstract}
Las dificultades de aprendizaje están definidas como reales por un complejo conjunto de prácticas legales y educativas y gobernadas por reglas y políticas escolares. Son objetos que están culturalmente construidos por las reglas de la escuela, sus leyes y sus prácticas educativas cotidianas (MEHAN; HERTWECK; MEIHIS, 1986, p. 85).
\end{abstract}

Así, el propio sistema educativo constituye "un mundo bien organizado para etiquetar y discapacitar" (MCDERMOTT, 1993, p. 274; VARENNE; MCDERMOTT, 1998, p. 42) a aquellos individuos que presentan dificultades para adaptarse sus reglas y no responden a las expectativas de las actividades institucionales organizadas en tales contextos.

\title{
3.1. Hacer visibles las Dificultades de Aprendizaje (To make evident Learning Disabilities)
}

La visibilidad de las Dificultades de A prendizaje requiere una serie de condiciones interaccionales y estructurales que actúan como "mecanismos de objetivación" (FOUCAULT, 1977, p. 187) haciendo sobresalir ciertas características individuales que marcan la deficiencia.

\begin{abstract}
La manifestación de las Dificultades de A prendizaje requiere un marco institucional que asigne determinados significados a la conducta de los estudiantes que, en otros contextos culturales, no supondrían la misma trascendencia. (DUDLEY-MARLING, 2004, p. 484).
\end{abstract}

Entre estos mecanismos, ocupan un lugar destacado las prácticas diagnósticas a través de las que se diferencia, juzga y clasifica a los alumnos, estableciendo para ellos determinadas expectativas de progreso. No obstante, existen otras prácticas que actúan como requisitos que propician la designación institucional de "la diferencia como deficiencia". 
Dudley-Marling (2004, p. 485) enumera una serie de requerimientos para que una persona sea considerada "inteligente"/ "Iista" en los contextos escolares. Siguiendo su mismo razonamiento pero en sentido inverso, para hacer visibles las Dificultades de A prendizaje en el contexto educativo es necesario que estén presentes una serie de condiciones: (a) en primer lugar, se requiere un conjunto de están dares culturales que definan a una persona como discapacitado/ deficiente; (b) oportunidades para demostrar la falta de competencia; (c) que éstas ocurran justo en el momento y el lugar adecuados; (c) por lo menos la presencia teórica de personas que desarrollen mejor las mismas tareas; y (d) la presencia de personas a las que se le ha concedido la autoridad de juzgar quién será incluido en esta categoría.

Un alumno con Dificultades de Aprendizaje se encuentra incluido dentro de la categoría más amplia "Alumno con Necesidades Educativas Especiales" (ACNEE) por presentar una serie de necesidades que requieren una atención diferenciada. Sin embargo, éstas NEE, resultado de una serie de dificultades en el aprendizaje, no son una característica individual, ya que el mismo contexto social forma parte activa en la determinación de qué se considera una "necesidad" y cuáles de ellas son "especiales".

Dyson (1990) afirma que las necesidades especiales no son objetivamente "reales" sino más bien un producto social, porque no permanecen estables a lo largo de diferentes contextos, sino que varían, e incluso desaparecen, en función de las circunstancias sociales (diferentes discursos, distintos tipos de escuela o de estructura social, con respecto al grupo de referencia, etc.). La reconceptualización delas NEE que supone este planteamiento conlleva asimismo una revisión del concepto de competencia, éxito y fracaso escolar más allá de las características individuales del alumnado.

\subsection{El CONCEPTO DE NORMATIVIDAD Y SUS VALORES ATRIBUID OS (CONCEPT OF NORMATIVITY AND ITS ATTRIBUTED VALUES)}

Si pretendemos llegar a comprender los significados que subyacen a estas prácticas institucional es es necesario explorar la significatividad de la idea de normatividad. La "normatividad" (GARLAND THOMAS, 1999) es socialmente producida y reproducida, así como los valores que se asignan a las variaciones con respecto a ella y que tienen que ver con la diversidad humana: "La desviación no existe independientemente de la comunidad de expertos que hacen el diagnóstico y llevan a cabo medidas especiales" (KIVINEN; KIVIRAUMA, 1988, p. 203).

La "norma", conforme Gimeno (2000), constituye una forma de homogeneización, subordinación y sometimiento legitimado de lo diferente a ciertos patrones unitarios que lo "normalicen". La valoración que se atribuye a una desviación en relación a la "norma” depende dela percepción y la orientación que adoptemos en referencia a la diversidad o diferencia. 
Como consecuencia de la hegemonía de la tradición individualista y del déficit, las variaciones de la norma son frecuentemente percibidas como limitaciones, discapacidades y patologías. Estas formas convencionales y naturalizadas de pensar y hablar acerca de la diferencia, están revestidas de presupuestos ideológicos aceptados como mero "sentido común", que determinan las prácticas y los significados atribuidos a estas variaciones así como su conceptualización (DUDLEY-MARLING; DIPPO, 1995; FAIRCLOUGH, 1989).

De acuerdo con estos planteamientos, el concepto de "Dificultades de A prendizaje", como categoría y construcción social, podría ser deconstruída descomponiéndola en un número designificados específicos y operacionales según indica Stangvik (1998, p. 144). Siguiendo la propuesta de Reid y Valle (2004), al analizar los significados sociales queseatribuyen a las variaciones en la diversidad humana es posible cuestionar las valoraciones implícitas o explícitas que construyen categorías binarias excluyentes (como normal-anormal; capazdiscapaz) y a su vez mutuamente constituyentes. Con lo cual, sería plausible cambiar la naturaleza de las "Dificultades de A prendizaje" 0 en definitiva de la "discapacidad" revisando nuestras concepciones sobre "capacidad", y la de "anormalidad" revisando nuestras definiciones de "normal"; es decir, cambiando nuestros discursos y prácticas discursivas. El mismo pensamiento dual ista se aplica a la identificación del éxito escolar con el esfuerzo y la habilidad, y del fracaso escolar con la deficiencia (DUDLEY-MARLING, 2004).

3.3. LA CONSTRUCCIÓN DEL DISCURSO ACERCA DE LA DIVERSIDAD: EL DISCURSO COMO PRÁCTICA SOCIAL (The CONSTRUCTION OF DISCOURSE ABOUT DIVERSITY: DISCOURSE AS SOCIAL PRACTICE)

El lenguaje no es simplemente un conjunto de signos cuya combinación nos permitecrear discursos para comunicarnos con otrosy compartir significados. Entrelas categorías queusamos y nuestra forma de percibir el mundo existe una circularidad. Esta característica supone que nuestros discursos (y las categorías que se incluyen en ellos) no solo describen la realidad social, sino que tienen la capacidad de conformarla, como "prácticas que sistemáticamente forman los objetos de los cuales hablan" (FOUCAULT, 1972, p. 49). Delimitar quién pertenece a una determinada categoría de individuos, como los alumnos con NEE o DA, depende de los términos que se están usando para definir dichos conceptos (BOOTH, 1998).

La evolución terminológica de los conceptos empleados en el ámbito educativo a lo largo de los años, muestra cómo han ido variado las categorías empleadas para describir a determi nados grupos dealumnos (como Discapacidad, Retraso Mental, Dificultades de A prendizaje, Necesidades Educativas Especiales, etc.) y cómo esta "formación y re-formación discursiva (p.e. cambios en las reglas que determinan qué se puede decir) conforma y reconfirma los conocimientos disciplinarios" queactualmenterigen la configuración del actual sistema educativo (FOUCAULT, 1972, apud REID; VALLE, 2004, p. 477). 
El uso decategorías en el contexto escolar sirvecomo un recurso retórico que crea un marco común para la comprensión de las dificultades dentro de la comunidad educativa (profesores, padres, y otros agentes implicados), es decir, hace posible la existencia de significados compartidos entre personas respecto a los objetos y hechos que identifican en términos estandarizados (GOODWIN; OPPER, 1992)

Mediante el lenguaje que usamos transmitimos los presupuestos que asumimos y que están presentes de manera implícita en las cuestiones que nos planteamos, en las interpretaciones que formulamos y las prácticas que realizamos. De esta forma, producimos y mantenemos las Dificultades de A prendizajey a la vez, éstas producen y mantienen su propio discurso, empleado al mismo tiempo en la organización de los mecanismos de apoyo y compensación educativa.

Considerando como decíamos que el conocimiento determina las prácticas, éstas determinan a su vez el conocimiento y así sucesivamente (FOUCAULT, 1972), nos encontramos ante un proceso circular difícil de romper en el que se establece una relación sistémica de interinfluencias entre procesos que tienen lugar a diferentes niveles. A nivel macro o institucional, el marco normativo que regula y articula los mecanismos de identificación, las actitudes sociales que conciben la diversidad como deficiencia y los patrones de actuación que sostienen estos valores en el ámbito escolar influyen en la estructura, las prácticas y las interacciones social es que tienen lugar a nivel micro en el contexto del aula, mientras éstas vienen a reforzar esta misma cultura de la diferencia.

Foucault propone el análisis tanto de los "discursos formales" a nivel macro como de las "prácticas discursivas" o "discursos informal es" que se establecen entre los participantes como forma de descubrir los "puntos de resistencia" (FOUCAULT, 1976, p. 95) en los que se observan las relaciones de poder que están mantenidas por significados sociales compartidos y reglas no explícitas que subyacen al funcionamiento grupal. Veamos un ejemplo de ello mediante un caso concreto.

\section{La construcción de categorías en la escuela. Estudio de un caso (The CONSTRUCTION OF CATEGORIES IN SCHOOL. A CASE STUDY)}

El ejemplo que se presenta a continuación, ha sido extraído de una investigación realizada en un Colegio Público de una ciudad de Madrid a través de un taller extraescolar en el que participaron 33 alumnos (23 niñas y 10 niños) de edades comprendidas entre 8 y 12 años y cuyo objetivo fundamental estaba relacionado con la alfabetización en los medios. El taller se realizó durante los meses de enero a marzo del curso 2003-2004 y constó de 12 sesiones, de aproximadamente dos horas de duración, realizadas semanalmente en el aula de informática del colegio al terminar el horario escolar. 
En este taller, "Periodistas", pretendíamos introducir en la escuela situaciones de la vida cotidiana que permitieran a los niños acercarse a la cultura local de su ciudad desde el punto de vista de las personas que participan en ella, utilizando para ello diferentes códigos comunicativos (oral, escrito y audiovisual) en un contexto en el queesta actividad adquiriese sentido y resultara significativa (ver SÁ NCHEZ; MÉNDEZ, 2006). Dividimos el taller en tres grupos en relación con los ámbitos de trabajo que establecimos y los subdividimos a su vez en otros más pequeños, de 2 a 3 niños.

Uno de los grupos estaba compuesto por dos niñas de 9 años, una de ellas con diagnóstico de "Necesidades Educativas Especiales asociadas a discapacidad" ("E"), y por una de las investigadoras (Investigadora "S".). Según nos informó el director del centro educativo, "E" presenta una patología conocida como hidrocefalia (por lo quea los pocos años de nacer se le implantó una válvula de derivación o by-pass) y una discapacidad motora. Según el informe al que pudimos acceder en la entrevista con su tutora, las consecuencias que se derivan del diagnóstico clínico para su aprendizaje tienen que ver con dificultades en el área de motricidad. Respecto a sus capacidades cognitivas, su nivel de competencia general es adecuado a los objetivos de su nivel, con unos resultados global es medio-bajo en las capaci dades cognitivas, inferiores en el área perceptivomanipulativa que en la verbal-numérica. La respuesta institucional a este diagnóstico consiste en la realización de adaptaciones curriculares significativas en las áreas en de Educación Física, Educación Artística, Lengua, Matemáticas y Conocimiento del medio. A simismo, "E" recibe apoyo externo al aula 2 veces por semana, fundamentalmente en matemáticas.

\subsection{Analizando las prácticas educativas desde el discurso pedagógico (Analysing EDUCATIVE PRACTICE FROM PEDAGOGIC DISCOURSE)}

Pasamos ahora a comentar algunos aspectos relacionados con la entrevista que realizamos a la tutora de "E" y en la que se hacen explícitos determinados aspectos del discurso implicados en la configuración de las categorías escolares.

Desde un punto de vista analítico, la entrevista puede verse como una serie de intentos de clasificación. Mientras la tutora describía las conductas de " $E$ ", la Investigadora " $L$ ", de manera inintencionada, buscaba en su discurso una coherencia que le permitiera interpretar y definir dichos comportamientos en correspondencia con la categoría que a "E" le había sido asignada.

Como vemos en el siguiente fragmento, cuando dice "Ios hay que son como "E" (220), la tutora se refiere a "E" en función de unas características grupales estereotipadas que se corresponden con las de los alumnos incluidos en la categoría de "Dificultades de Aprendizaje". Como ya hemos mencionado, estas categorías tienen un determinado valor social en comparación con otros 
grupos: en este caso, "Ios que no son como "E". A pesar de que se observa que la conducta de los iguales hace que "E" sufra un rechazo, una limitación y en ocasiones negación de su participación, su tutora afirma que " $E$ " "no tiene problemas de relación" (220) porque no es agresiva, según ella "socialmente muy bien" (220).

Durante el proceso dela entrevista, observamos que la tutora siempre busca la causa de las dificultades o los límites (56) en el propio alumno, sin dirigir la atención al contexto donde estas conductas se producen, sin contextual izarlas en relación a otros hechos concretos ocurridos en clase que puedan ser relevantes para su comprensión. En ningún momento se realiza un análisis de las circunstancias en que surgen este tipo de dificultades que pueda ayudar a una mejor comprensión de características comunes a todas ellas, no se cuestiona si la organización del contexto o la interacción social pueden estar limitando la participación y el aprendizajede "E". Sencillamente, no sebuscan interpretaciones alternativas queconsi deren estos aspectos como determinantes en las Dificultades de A prendizaje, y más aún cuando éstas pueden atribuirse a un diagnóstico y argumentarse mediante el discurso psicomédico, como en el caso de "E".

\subsubsection{Expectativas respecto a la eVolución de "E" (Expectations about “E" progress)}

Para su tutora, "E" ha madurado, empieza a ser realista y a darse cuenta de sus propias limitaciones (56), ya sabe que ella no va a poder ser como los otros, no va a poder alcanzar su nivel de competencia, sin embargo ha sido precisamente el hecho de no percibir este limite, de no dejarse llevar por las expectativas creadas por su condición, el que le ha permitido avanzar en su desarrollo, lanzarse y afrontar nuevos retos (56)

Es una niña que nunca se ha estancado (234), que insiste (103), no abandona, tiene afán de logro (105) y, sin embargo, pocas veces ha visto reconocido su esfuerzo constante con una calificación alta (103), como sus compañeros.

Todo el peso de las Dificultades de A prendizaje recae sobre "E" y sus capacidades limitadas. No se cuestiona el papel de la estructura organizacional del aula, los patrones de interacción entre los iguales, y otros elementos que están mediando el aprendizaje y la ejecución de "E". Como decíamos, la categorización, o la valoración que se hace de los alumnos mediante los informes escolares, constituye un recurso institucional fundamental en la toma de decisiones que determina el tipo de medidas de apoyo que va a recibir "E", las áreas en las que requiere una adaptación curricular y otro tipo de decisiones institucionales que "ayudan a "E" a adaptarse al sistema educativo".

Los protocolos y actuaciones pedagógicas se encuentran ya predefinidas o definidas a priori de las características individuales del niño. Una vez determinadas las medidas asignadas institucionalmente en el tratamiento de 
las dificultades de "E", a partir de su diagnóstico y la categoría correspondiente, no existe una discusión pedagógica acerca de las consecuencias que éstas están teniendo en el aprendizaje y en el desarrollo de "E", solo existe un seguimiento curricular como consecuencia de un concepto de aprendizaje limitado a la adquisición de contenidos y habilidades.

\section{Conclusiones (Concluding remarks)}

La percepción de la diferencia como desviación social, deficiencia, limitación o fal ta de competencia y las actitudes hacia la diversidad que generan dichas concepciones siguen prevaleciendo en el ámbito escolar, constituyendo el sustrato sobre el que se asienta la construcción social de las de Dificultades de A prendizaje y los mecanismos de exclusión encubierta, legitimados por el discurso institucional y las decisiones y prácticas pedagógicas establecidas en base a él.

Los procesos que están generando y manteniendo estas condiciones de desigualdad tienen su base en una determinada conceptualización, en este caso, de las Dificultades de A prendizaje, construida en base a convicciones, reglas constitutivas y significados sociales creados en la comunidad educativa y compartidos por todos sus miembros. Por ello, para producir un cambio en la dinámica del grupo no podemos incidir en los individuos de forma aislada, es necesario modificar todos los el ementos que configuran la estructura de la actividad.

La integración escolar en sí misma no es capaz de mejorar el estatus social delos al umnos "especiales", de ahí la necesidad establecer mecanismos capaces de incidir en la dinámica del grupo de iguales con el objetivo de generar cambios en los roles y en el estatus social que ocupan estos alumnos en él, a través de la transformación de su participación en la actividad sociocultural del grupo (LAVE; WEN GER, 1991; REID; VALLEY, 2004; RUEDA et al., 2000). Negociando Ios esquemas tipificadores en la interacción social y las prácticas a las que están vinculadas los roles y el estatus de los individuos, será más probable modificar las concepciones acerca de la diversidad y las prácticas que se derivan de ellas.

Empleando la terminología deStangvik, estos cambios en la naturaleza de la interacción entre los participantes y en la cultura del grupo solo podrán mantenerse, si se establece una continuidad y una actuación complementaria, entre los diferentes marcos de conocimiento y niveles de la escuela como institución, de modo que los distintos discursos, paradigmas, modelos, teorías y metateorías, prácticas e instrumentos presentes en la institución y la comunidad escolar no sean contradictorios. 
SÁNCHEZ, S.

\section{Referencias}

ARMSTRONG, D.; ARMSTRONG, F.;BARTON , L. From theory to practice: Special education and thesocial relation for academic production. In: CLARK, C.; DYSON, A.; MILLWA RD, A. (Eds.). Theorising special education. London: Routhledge, 1998. p. 32-41.

BECKER, H. O utsiders: studies in thesociology of deviance. New York: TheFreePress, 1963.

BERGER, P. L.; LUCKMANN , T. La construcción social dela realidad. BuenosAires: Amorrortu Editores, 1968.

BOOTH, T. Thepoverty of special education: Theories to therescue? In: CLARK, C.; DYSON, A.; MILLWARD, A. (Eds.). Theorising special education. London: Routhledge, 1998. p. 79-89.

CLARK, C.; DYSON , A.; MILLWARD, A Introducing theissue of theorising. In: CLARK, C.; DYSON, A.; MILLWARD, A. (Eds.). Theorising special education. London: Routhledge, 1998. p. 1-6.

COLE, M. Can cultural psychology help us think about diversity? M ind, Cultureand A ctivity, v. 5, n. 4, p. 291-304, 1998.

DÍAZ-A GUADO, M.J.; MARTINEZ, R.; MARTIN , G. La evaluación delasactitudes hacia la violencia y la diversidad. In: DÍAZ-AGUADO, M. J.; MARTINEZ, R.; MARTIN, G. (Eds.). Prevención dela violencia y lucha contra la exclusión desdela adolescencia. La viol encia entreiguales en la escuela y en el ocio. Estudios comparativos e instrumentos de evaluación. Madrid: Ministerio deTrabajo y A suntos Social es. Injueve, 2004. p. 89-125. v. 1.

DUDLEY-MARLING, C. The social construction of learning disabilities. Journal of Learning Disabilities, v. 37, n. 6, p. 482-489, N ovember/ December, 2004.

DUDLEY-MARLING, C.; DIPPO, D. What learning disability does: sustaining the ideology of schooling. J ournal of Learning D isabilities, v. 28, p. 406-414, 1995.

FAIRCLOUGH, N. Language and power. London: Longman, 1989.

FOUCAULT, M. Thearcheology of knowledgeand thediscourseon language. New York: Pantheon, 1972.

GARLAND THOM SON, R. Thenew disability studies: Inclusion or tolerance? A D FL Bulletin, New York, A ssociation of Departments of Foreign Languages, v. 31, n.1, p. 49-53, Fall 1999.

GERGEN, K.J. Social understanding and theinscription of self. In: STIGLER, J. W.; SHWEDER, R. A.; HERDT, G. S. (Eds.). Cultural psychology: essays on comparativehuman development. New York: CambridgeUniversity Press, 1990. p. 569-606.

GIMENO, J. Edu car y convivir en la cultura global. 2. ed. Madrid: Morata, 2002.

GINDIS, B. Remedation trough education: Sociocultural theory and children with special needs. In:KOZULIN , A.; GINDIS, B.; A GEYEV, V. S.; MILLER, S. M. (Eds.). V ygotsky's educational theory in cultural context. Cambridge: CambridgeUniversity Press, 2003. p. 200-221.

DURANTI, A.; GOODWIN, C. Rethinking context: an introduction. In: DURANTI, A.; GOODWIN, C. (Eds.). R ethinking context. Languageas an interactive phenomen on. Cambridge, England: CambridgeUniversity Press, 1992. p.1-42. 
GUTIÉRREZ, K. D.; BA QUEDAN O-LÓPEZ, P.; TEJEDA, C. Rethinking diversity: Hybridity language practices in the third space. $M$ ind, Culture and A ctivity, Cambridge MA, Cambridge U niversity Press, v. 6, n. 4, p. 286-303, 1999.

GUTIÉRREZ, Kris D. Studying cultural practices in urban learning communities. H uman D evelopment, v. 45, n. 4, p. 312-321, 2002.

HALL, S. (Ed.). Representation: cultural representations and signifying practices. London: Sage, 2000.

HJÖRNE, E.; SÄLJÖ, R. “There is something about Julia”. Symptonms, categories, and the process of invoquin ADHD in the Swedish school: a casestudy. J ournal of Language, Identity, and Education, Mahwah NJ, v. 3, n. 1, p. 1-24, 2004.

LAVE, J.; WENGER, E. Situated learning. Legitimate peripheral participation. Cambridge: University of CambridgePress, 1991.

MÄKITALO, Å.; SÄLJÖ, R. Talk in institutional context and institutional context in talk: Categories as situated practices. Text, v. 22, n. 1, p. 57-82, 2002.

MCDERMOTT, R. J. The acquisition of a child by a learning disability. In: CHAIKLIN, S.; LAVE, J. (Eds.). U nderstan ding practice: focus on activity and context. N ew York: Cambridge University Press, 1993. p. 69-305.

MCDERMOTT, R.J.; VAREN NE, H. Culture, development, disability. In:JESSOR, R.; COLBY, A.; SHWEDER, R. A. (Eds.). Ethnography and human devel opment. Context and meaning in social inquiry. Chicago \& London: TheUniversity of Chicago Press, 1996. p. 101-126.

MCDERMOTT, R.J.; VAREN NE, H. A dam, A dam, A dam, and Adam: thecultural construction of a Learning Disability. In: VAREN NE, H.; M CDERMOTT, R. J. (Eds.). Successful failure: the school A merica builds. Boulder, CO: Westview Press, 1998. p. 25-44.

MEHAN, H.; HERTWECK, A.; MEIHLS, J. L. H andicapping thehandicapped. Palo Alto: Stanford University Press, 1986.

MOLL, L. C. Vygotski, la educación y la cultura en acción. In: ÁLVAREZ, A. (Ed.). H acia un currículum cultural. La vigencia de V ygotski en la educación. Madrid: Fundación Infancia y A prendizaje, 1997. p. 39-53.

RITZER, G. Teoría sociológica clásica. Madrid: McGraw-Hill, 1993.

RUEDA, R.; GA LLEGO, M. A.; MOLL, L. C. The Least RestrictiveEnvironment. A placeor a context? Remedial and Special Education, v. 21, n. 2, p. 70-78, 2000.

SÁNCHEZ VALVERDE, S.; MÉNDEZ ZABALLOS, L. Diversidad e inclusión: Sandra se hace escritora. In: LACASA, P. (Ed). A prendiendo periodismo digital. Historias de pequeñas escritoras. Madrid: Visor, 2006. p. 179-207.

SCHÜTZ, A. La construcción significativa del mundo social: Introducción a la sociología comprensiva. Barcelona: Paidós, 1993.

SKRTIC, T. M. D isability and democracy: Reconstructing (special) education for postmodernity. New York: Teachers CollegePress, 1995.

STANGVIK, G. Conflicting perspectives on learning disabilities. In: CLARK, C., DYSON , A.; MILLWARD, A. (Eds.). Theorising special education. London: Routhledge, 1998. p.137-155. 
SÁNCHEZ, $S$.

VYGOTSKY, L. S. The collected works of I. S. V ygotsky. The fundamentals of defectology (abnormal psychology and learning disabilities). New York: Plenum Press, 1993. v. 2.

WEN GER, E. Comunidades depráctica. aprendizaje, significado eidentidad. Barcelona: Paidós, 2001.

WERTSCH, J. V. V oices of the mind: a sociocultural approach to mediated action. Cambridge: Harvard University Press, 1991.

WERTSCH, J. V. M ind as action. N ew York, NY: Oxford University Press, 1998. 